\title{
CONFIANZA EN LAS INSTITUCIONES ELECTORALES EN MÉXICO: EL IFE BAJO LA MIRADA CIUDADANA
}

Leticia Heras Gómez

\begin{abstract}
Resumen
La confianza ciudadana en las instituciones políticas es parte insoslayable de la agenda de la democratización de los países que recién factura democrática. Sin embargo, los niveles de confianza que se han alcanzado en países como México no parecen avanzar con el ritmo necesario para dar una mayor estabilidad y duración a las instituciones políticas representativas de la democracia, un caso de suma importancia en este contexto es el Instituto Federal Electoral (IFE) en México. El propósito del artículo es examinar cual ha sido la evolución en la confianza de los mexicanos, tanto interpersonal como hacia ésta institución en la primera década del siglo. El análisis se basa en los datos de las encuestas de Latin American Public Opinion Project (LAPOP) y de World Values Survey en las rondas correspondientes a dicho decenio; así como en resultados de la empresa Mitofsky y en las ENCUP 2001, 2003, 2005, 2008. El argumento central es que el desarrollo de la confianza interpersonal aún exhibe signos precarios para soportar el edificio de una democracia en construcción y que ello restringe la formación de redes ciudadanas que den cuenta de un capital social en desarrollo, que otorgue solidez a la incipiente democracia. Pero sin embargo, la confianza hacia el IFE si muestra una tendencia positiva ascendente, lo que es una buena noticia para la democracia mexicana, al menos en las primicias de la siguiente elección presidencial.
\end{abstract}

Palabras claves: Confianza; Institución; Democracia; Democratización.

\begin{abstract}
Confidence in political institutions can be a substantial component of democratization agenda's, mainly in countries where democracy is still at early stages. However, levels of confidence reached in countries like Mexico do not seem to advance in order to bestow stability and permanence to political institutions, which is the case of the Federal Electoral Institute (IFE). The purpose of this essay is to examine the evolution of confidence among Mexicans, both interpersonal and institutional towards the IFE, in the first decade of the century. The analysis is based upon Latin American Public Opinion Project (LAPOP) and in the World Values Survey, taken the rounds of such decade; as well as Data from Mitofsky Consulting and Encuesta Nacional de Cultura Política (ENCUP) surveys from 2001, 2003, 2005, 2008 years. The main argument is that in Mexico interpersonal confidence still shows precarious levels therefore citizen networks are not developing social capital, which in turn would provide endurance to political institutions. Nevertheless confidence in the IFE shows a positive increase, and that is the good news to Mexican democracy.
\end{abstract}

Keywords: Confidence; Institution; Democracy; Democratization. 


\section{Introducción}

El propósito del artículo es examinar cual ha sido la evolución en la confianza de los mexicanos, tanto interpersonal como hacia ésta institución en la primera década del siglo. El análisis se basa en los datos de las encuestas de Latin American Public Opinion Project (LAPOP) y de Encuesta Mundial de Valores en las rondas correspondientes a dicho decenio. Y para el caso del IFE (Instituto Federal Electoral) se utilizan los datos de Parametría, Reforma, ENCUP 1 Y ENCUP 2, Instituto de Investigaciones Sociales- UNAM, Ipsos Marketing Research y Consulta Mitofsky; a largo de 10 años, desde 1999 a 2010.

Existe un acuerdo más o menos común (COLEMAN, 1988, 1990; PUTNAM, 1993，2000; INGLEHART，1988; INGLEHART y WELZEL, 2005) de que la confianza interpersonal y social constituye uno de los cimientos más importantes en la edificación del sistema democrático, y que sin este ingrediente resulta muy penosa - y no carente de retrocesos - la implantación y desarrollo de las instituciones políticas y los tejidos sociales característicos de dicho sistema.

De ahí, que resulte de suma relevancia seguir la trayectoria que esa confianza social e interpersonal ha tenido en México, a partir de la instalación formal de la democracia en el país, acudiendo a un esquema de análisis comparativo regional y nacional histórico.

Vale la pena mencionar, de todos modos, que el tema de la confianza aún se encuentra en amplia discusión y que no debe tomarse como un concepto inamovible (en todo caso no existe tal cosa), pero que estudios como el presente pueden contribuir a confrontar su validez teórica; en virtud de que ponemos en operación empírica los distintos elementos que lo componen.

Asumimos que la confianza interpersonal y social, sin bien son componentes esenciales de la vida democrática, la formación de éstos obedece tanto al desarrollo de una cultura democrática, como a los esfuerzos institucionales por procurar ganar y generar dicha confianza entre la misma ciudadanía. Y que para el caso de México los valores de confianza interpersonal se encuentran en un nivel medio bajo respecto al resto de los países del continente, lo cual indica que aún no se construyen redes sociales sólidas deseables para la plena instalación democrática. Sin embargo, los datos sobre confianza en las instituciones electorales muestran signos positivos ascendentes, los cuales apuntan a un mayor asentamiento de la confianza como valor democrático y como parte de la legitimidad de las instituciones políticas.

Confianza y accountability como pilares de la democracia, merecen ser puestos a prueba en un ejercicio comparativo, de forma tal que se pueda apreciar su avance y consistencia, al menos en un periodo de tiempo que permita su apreciación. Dedicamos el presente artículo al tema de la 
confianza, pues ya en otros espacios hemos analizado el problema de la accountability con mayor detenimiento (HERAS, 2008).

El artículo está dividido en dos partes. La primera se dedica a examinar las más recientes aportaciones teóricas al debate de la confianza interpersonal y social. La segunda es un intento por evaluar hasta que grado se ha instalado ya el esquema de confianza en México, en términos históricos en concreto hacia la principal institución electoral de México que es el IFE.

De acuerdo a los datos analizados parece claro que México no está a la vanguardia en los procesos de generación de confianza interpersonal y que sus vecinos del sur llevan la delantera. En cambio la confianza hacia las instituciones lectorales parece mostrar signos positivos. Estudiamos ambos procesos a la luz de los datos cuantitativos disponibles.

\section{Confianza interpersonal y social: una recapitulación conceptual}

Para abordar el tema de la confianza se requiere puntualizar dos de sus fuentes principales: la noción de capital social y su acepción como valor democrático.

En el primer caso la confianza interpersonal y social es uno de los tres componentes del capital social, en sus más conocidas versiones (COLEMAN, 1988, 1990; PUTNAM, 1993, 2000; LIN, 2001) junto con las redes y las normas. En principio la democracia para su plena instalación demanda que los ciudadanos experimenten una gran confianza en términos personales, con el vecino, el colega del trabajo, el amigo o la familia, etc. y que esta confianza se extienda a la vida pública; es decir, del ciudadano hacia sus autoridades, sus instituciones y en todas sus relaciones sociales. A la primera se le conoce como confianza interpersonal y a la segunda como confianza social.

En su conjunto el concepto de Capital Social incluye aspectos de confianza, asociatividad, conciencia cívica y valores éticos predominantes pueden facilitar el desarrollo socio-político de una determinada sociedad.

En el caso de la confianza social en la medida que los individuos experimentan confiabilidad en sus relaciones sociales, pueden formar asociaciones, redes, organizaciones, que a su vez se establecen como frentes ciudadanos exigentes de un ejercicio legítimo y transparente de la autoridad. $Y$ de modo inverso un cuerpo de autoridades que goza de la confianza de los ciudadanos, lleva a cabo sus funciones con apego más estricto a las normas y con miras a mejorar continuamente su desempeño.

En general las sociedades no democráticas experimentan un bajo nivel de confianza interpersonal y social; y en aquellas de democracia consolidada la presencia de la confianza permite un extenso desarrollo de la vida asociativa (llamada también democracia asociativa). Ello debido a que la confianza reduce los costos de transacción en la vida social y facilita acciones colectivas y de cooperación (PUTMAN, 1993). El desarrollo de la confianza, y la consecuente formación de redes, permite que los individuos ahorren los recursos y las energías, que les representaría su acción en forma individual. 
De ahí que en el esquema democrático la confianza sea un activo tanto para la participación organizada de la sociedad, como para la respuesta de la autoridad.

En el segundo caso, la confianza como valor democrático ha sido especialmente importante para aquellos países que construyen hoy en día el edificio democrático. En primer lugar porque forma parte del catálogo de valores mediante los cuales es posible cimentar sus instituciones. Hasta ahora y aún en su acepción límite que es la definición de Churchill, la democracia requiere de la confianza al interior de la polity, como una base social para la solución pacífica de conflictos, pues lo contrario entorpece la vida asociativa. El valor de la confianza es en buena medida un signo de salud de una comunidad política.

En términos latos, dice Noemí Luján Ponce "la confianza se define como una expectativa o un apuesta del sujeto que confía (truster) en el depositario de su confianza (trusted), que puede ser otra persona, un objeto, un institución, un valor, etc." (LUJÁN PONCE, 2009, p. 234) ${ }^{1}$. Ambas en español significan una relación social saludable para toda comunidad política, en virtud de la reciprocidad y certeza que representan para sus miembros (LUJÁN PONCE, 2009).

Ha sido Robert Putnam (1993, 2000 y 2004) quien mejor ha establecido la relación entre confianza y democracia. Ambas dimensiones colocadas dentro del concepto de capital social. El capital social "se refiere a las características de organización social, tales como la confianza, las normas y redes, que pueden mejorar la eficiencia de la sociedad mediante la facilitación de las acciones coordinadas" (PUTNAM, 1993, p. 38).

Dichas acciones coordinadas estimulan la creación de asociaciones, frentes, foros, organizaciones, que de toda índole pueden apuntalar la acción ciudadana en una democracia. En el fondo que un individuo pertenezca o busca formar una asociación tiene que ver con la posibilidad de aminorar los costos que le representaría actuar por su propia cuenta. Pero para ello necesita poder confiar en los otros, esto es en aquellos con intereses similares. Así la confianza es la pieza clave para la organización ciudadana, la confianza "estabiliza vínculos porque permite cálculos sobre el comportamiento de los otros" (MILLÁN y GORDON, 2004, p. 725).

En términos políticos este proceso opera en los dos sentidos: del ciudadano hacia la autoridad y de ésta al ciudadano, es un movimiento recíproco. De manera que si un solo polo no tiene la suficiente garantía de confiabilidad, seguramente el otro polo disminuirá su confianza. Por ello en una democracia incipiente, los niveles de confianza son siempre precarios, de ambas partes de la ecuación.

\footnotetext{
${ }^{1}$ La misma autora recupera de Claus Offe la distinción en Inglés que no existe en Español, entre trust y confidence. La primera con un mayor fundamento cognitivo y la segunda más parecida la fé (LUJÁN PONCE, 2009).
} 
Un aspecto importante en el desarrollo de la confianza es el tiempo en el que ésta se va implantando en una comunidad política. En una democracia dos elementos pueden generar su desarrollo: el mejoramiento del desempeño gubernamental y la proliferación de redes ciudadanas preocupadas por los asuntos de carácter público. Ambos demandan de plazos a distinto nivel y velocidad. El primero es más contingente y puede ser producido por eventos coyunturales en donde se muestre un más acertado trabajo gubernamental. Ejemplo de ello puede ser el cumplimiento de los ofrecimientos de campaña por aquellos que llegan al poder público y realizan lo prometido. Pero asimismo, hechos de corrupción alteran los niveles de confianza, y puede ser de manera abrupta.

El segundo, es decir el tiempo que toman las redes o asociaciones en transformar sus lazos de intercambio inicial por lazos formales de cooperación, puede tomar un largo tiempo, suele ser más sólido y no necesariamente es coyuntural. Ese largo plazo a su vez representa para la democracia una posibilidad de mayor duración.

De modo contrario, hay que decir que la desconfianza puede provenir de un pobre y deshonesto desempeño de las autoridades de todos los niveles de gobierno, y de una excesiva cautela en las relaciones interpersonales de los individuos. "En un contexto de desconfianza, los individuos se retiran a lo privado y expresan actitudes de apatía y cinismo hacia los asuntos públicos. Este individualismo 'amoral', destruye las instituciones y cancela cualquier posibilidad de cooperación" (LUJÁN PONCE, 2009, p. 234, subrayado por el autor). Justo este es el problema para la democracia: que la desconfianza disminuye los niveles de reciprocidad ciudadana y no permite que las instituciones típicas del sistema democrático se construyan sobre una base sólida. Tal es el caso de los sistemas electorales y de partidos, los congresos y los poderes ejecutivo y judicial.

Con esto último pasamos ahora a analizar los datos de confianza de México en términos comparativos. Antes, vale la pena dos críticas al tema de la confianza, como parte del capital social. Pues hay que reconocer que aunque en principio forma parte de los cimientos de los sistemas democráticos modernos, en la actualidad - es decir en esta primera década del siglo XXI - la confianza y la cooperación también aparecen en un tipo de asociaciones como las del crimen organizado, o en la red de relaciones de corrupción en las altas esferas de los mandos policiales y hasta gubernamentales las cuales habría que estudiar con detenimiento, pues pudieran estar basadas también en la estrecha y recíproca confiabilidad entre sus miembros. El antecedente que se ha hecho más famoso es el de la mafia en los Estados Unidos a principios del siglo XX. Aunque también el propio Putnam reconocía en un artículo reciente el peligro que representaba la formación de redes basadas en la confianza y la reciprocidad, cuyos resultados pueden tener externalidades o fines negativos ${ }^{2}$.

2 Putnam agregaba que en el caso de la red de individuos que hicieron estallar una bomba en un callejón en Oklahoma city era un ejemplo claro de capital social con una finalidad genuinamente

REVISTA DEBATES, Porto Alegre, v.5, n.1, p. 9-23, jan.-jun. 2011. 
Otra crítica relevante es que la investigación o más concretamente la reunión de los datos empíricos sobre confianza y sus posibilidades para conformarse en capital social requiere de series históricas, que no están disponibles en la medida que el investigador necesitaría para demostrar sus hipótesis. Este es el caso de los países latinoamericanos, en donde las encuestas que incluyen temas de confianza son relativamente recientes, de fines años ochenta. Y con más nivel de confianza datan de los años noventa. De manera que lo que podemos alegar en torno a la implantación de la confianza como valor democrático y como componente del capital social es una aproximación a la realidad y no una demostración de la misma.

Por tanto, ubicar los niveles que ha alcanzado la confianza en las instituciones en México, será una aproximación fundamentada en datos confiables de las encuestas disponibles de los últimos 10 años. Cabe aclarar que en el caso de México, el estudio sobre la evolución de la confianza es sumamente complicado. Las rondas de la ENCUP (Encuesta Nacional sobre Cultura Política y Prácticas Ciudadanas) que registran resultados sobre confianza son incomparables entre si por dos razones. Primero porque reflejan valores absolutos, y segundo porque el tipo de preguntas y respuestas difieren en cada ronda. Las dos primeras (2001 y 2003) pedían al entrevistado contestar si confiaban poco o mucho en las instituciones (SEGOB, 2001 y 2003). La encuesta del 2005 preguntaba sobre confianza en una escala de 1 a 10, cuya equivalencia con los valores de poco o mucho no es estrictamente confiable (SEGOB, 2005). Y la del 2008 solicitaba respuestas de confía mucho o confía algo (SEGOB, 2008). En consecuencia no se puede analizar una evolución con datos que son inconsistentes y las ENCUP en México, desafortunadamente, no nos ayudaron para el objetivo de este ensayo. En cambio y a pesar de que no gocen de amplio reconocimiento académico, los resultados de la consulta anual (o semestral) de la empresa privada Mitofsky (2010) pueden ser de mayor utilidad para establecer la evolución de la confianza en las instituciones políticas en México.

De todos modos comenzamos con una aproximación comparada en América latina y el mundo basada en los datos sobre confianza interpersonal que proporciona LAPOP (2008) y la Encuesta Mundial de Valores (EMV, 2008); para enseguida estudiar de manera concreta la confianza en las instituciones en México.

\section{Avance, estancamiento $o$ retroceso de la confianza en las instituciones políticas mexicanas}

Comenzamos con una gráfica (1) que nos muestra que en México el $57,3 \%$ de los mexicanos dice tener confianza en la gente de su comunidad. Ello significa que menos de 6 ciudadanos confía en su vecino, colega de

destructiva y agregaba que "todas las formas de capital social [...] pueden ser utilizadas para fines que son en algunas circunstancias destructivos" (PUTNAM, 1993, p. 3). 
trabajo o repartidor que llega a su casa. Y que aún la confianza interpersonal se encuentra en un nivel muy precario para formar redes o asociaciones que den fuerza al capital social, o que puedan apuntalar el valor en las nuevas generaciones.

Hay que notar que la desconfianza interpersonal en América Latina tiene raíces muy profundas que provienen de la herencia española hacia las culturas mesoamericanas, y que ésta se ha prolongado a través de las sucesivas invasiones extranjeras. Los países de la región no nacieron en un esquema de confianza entre sus habitantes, pues existían al menos dos culturas distintas: la criolla y la indígena; y en ningún caso la confianza fue un signo cultural fuertemente arraigado entre ambas. La historia de los siglos posteriores tampoco nos muestra que dicho valor se halla arraigado en las poblaciones mestizas. Durante el turbulento siglo XX, tampoco se fomentó desde los poderes públicos o se construyó desde la gente un piso de confianza interpersonal que ayudara a conformar redes, y por lo tanto a la llegada del siglo XXI, estamos a medio camino.

Otros países, sin embargo, cuentan ya con niveles de confianza más prometedores para la democracia. Exceptuando los de origen anglosajón, en América Latina, Costa Rica quizá sea el mejor ejemplo de confianza interpersonal solo por debajo de Canadá, pero aún arriba de los Estados Unidos. Sería posible afirmar que por este solo dato, un retroceso democrático en Costa Rica sería prácticamente improbable. 
Gráfica 1 - Confianza interpersonal en perspectiva comparada, 2008.

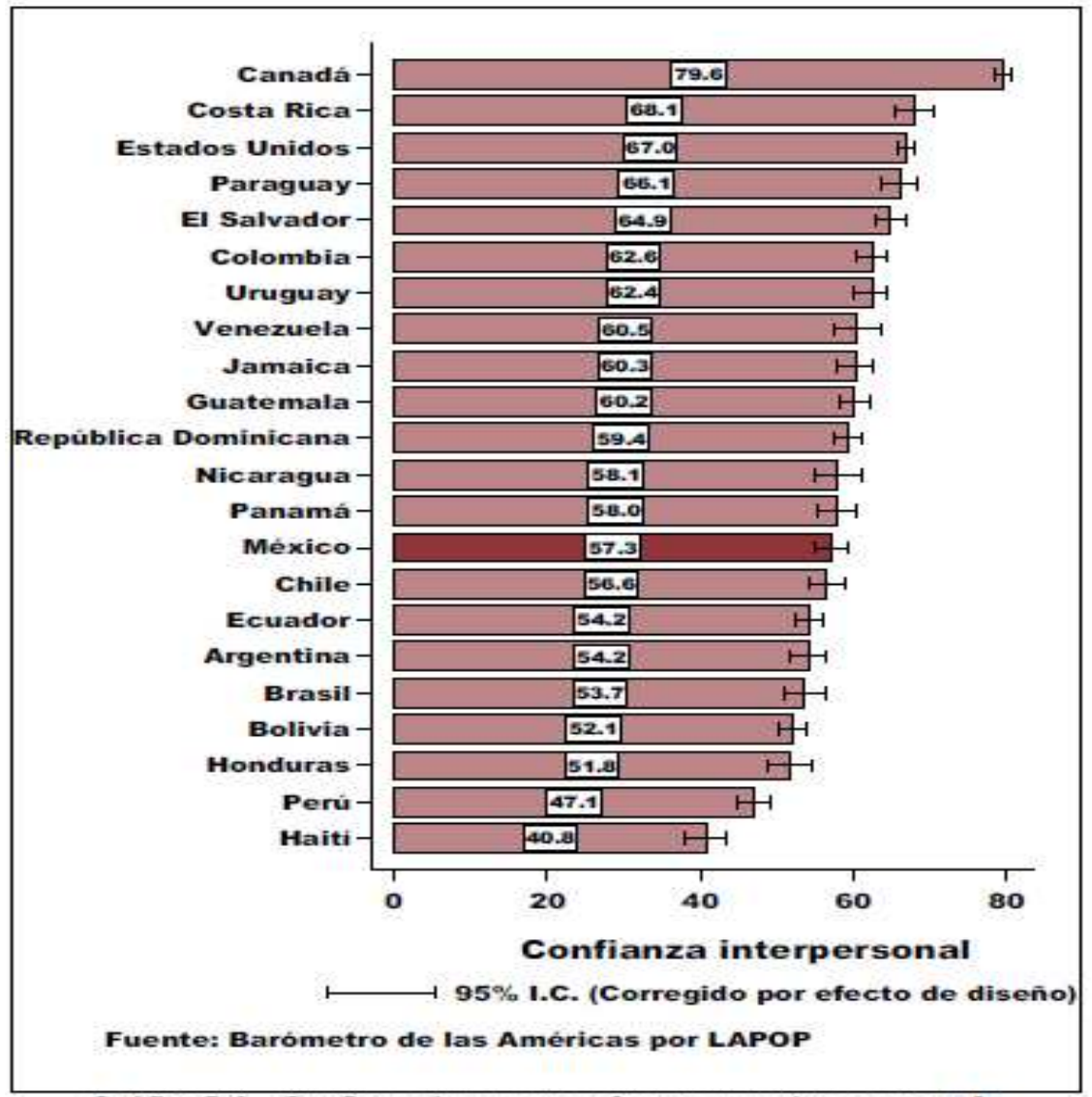

Gráfico I-6. Confianza interpersonal en perspectiva comparada

Como decíamos en la primera parte la confianza interpersonal es un proceso cultural de largo plazo, forma parte del tejido social que los individuos van formando a su alrededor. La democracia necesita de este factor, tanto para fortalecer la vida asociativa como para incentivar la confianza en las instituciones políticas.

Para el 2005, año en que se llevó a cabo la tercera Encuesta Nacional de Cultura Política (SEGOB, 2005) se preguntó a los ciudadanos entrevistados ¿Qué tanto pueden confiar en las demás personas? y, de acuerdo con los datos obtenidos, 6 de cada 10 ciudadanos dijeron confiar poco (64\%) en sus semejantes, $18 \%$ manifestaron no confiar nada y el restante $16 \%$ dijeron confiar mucho. Esto significa que la confianza interpersonal no se ha incrementado en el último lustro. $Y$ no es un dato positivo para la democracia en el país, pues aleja o al menos detiene la formación de capital social, como factor de consolidación de la democracia asociativa. 
La credibilidad en las instituciones electorales en México depende más del contexto político dentro del cual se llevan a cabo los procesos electorales, que de la propia institucionalidad alcanzada por el IFE y los institutos locales. Entendiendo por institucionalidad el arraigo y confianza que estos han ido desarrollando entre la población, y la calidad de su desempeño a la hora de organizar los comicios y de rendir cuentas en todos los aspectos del proceso.

Una forma de conocer dicho arraigo y el nivel de confianza es evaluar el IFE mediante las encuestas que lo ponen a prueba de cara a la población. Por ello reunimos todos los datos disponibles hasta ahora con respecto a la opinión de la población sobre el IFE, a saber: Parametría, Reforma, ENCUP 1 Y ENCUP 2, Instituto de Investigaciones Sociales- UNAM, Ipsos Marketing Research.

En las gráficas 2 y 3 podemos observar de manera comparativa los niveles de confianza de diversas instituciones en México. Con una diferencia más o menos significativa, entre creer y confiar, los mexicanos dicen confiar en un $37 \%$ en el IFE, y creer solamente un $19 \%$

Gráfica 2 - México: confianza ciudadana en instituciones, $2003^{3}(\%)$.

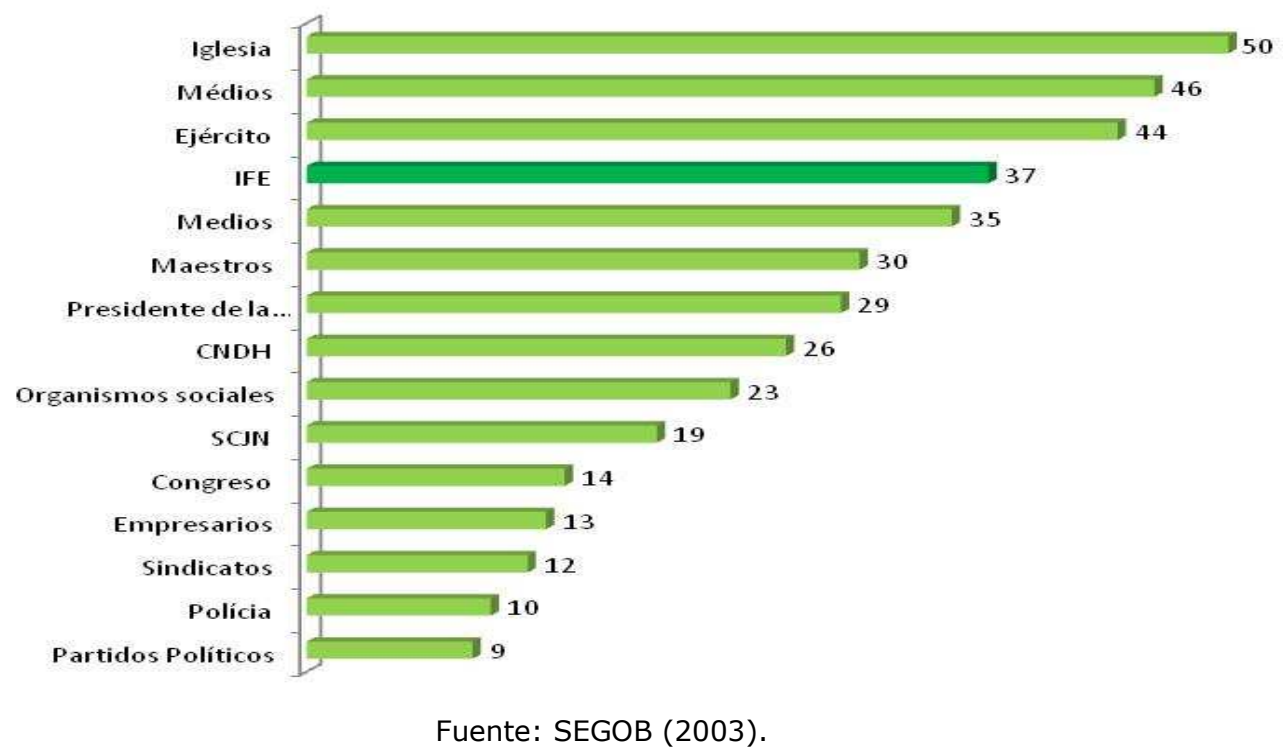

\footnotetext{
${ }^{3}$ Pregunta: En una escala en donde 0 es nada y 10 es mucho, qué tanto confía en las siguientes instituciones.
}

REVISTA DEBATES, Porto Alegre, v.5, n.1, p. 9-23, jan.-jun. 2011. 


\section{DOSSIÊ CAPITAL SOCIAL E COMPORTAMENTO ELEITORAL}

Gráfica 3 - México: credibilidad ciudadana en diferentes instituciones, $2003^{4}(\%)$.

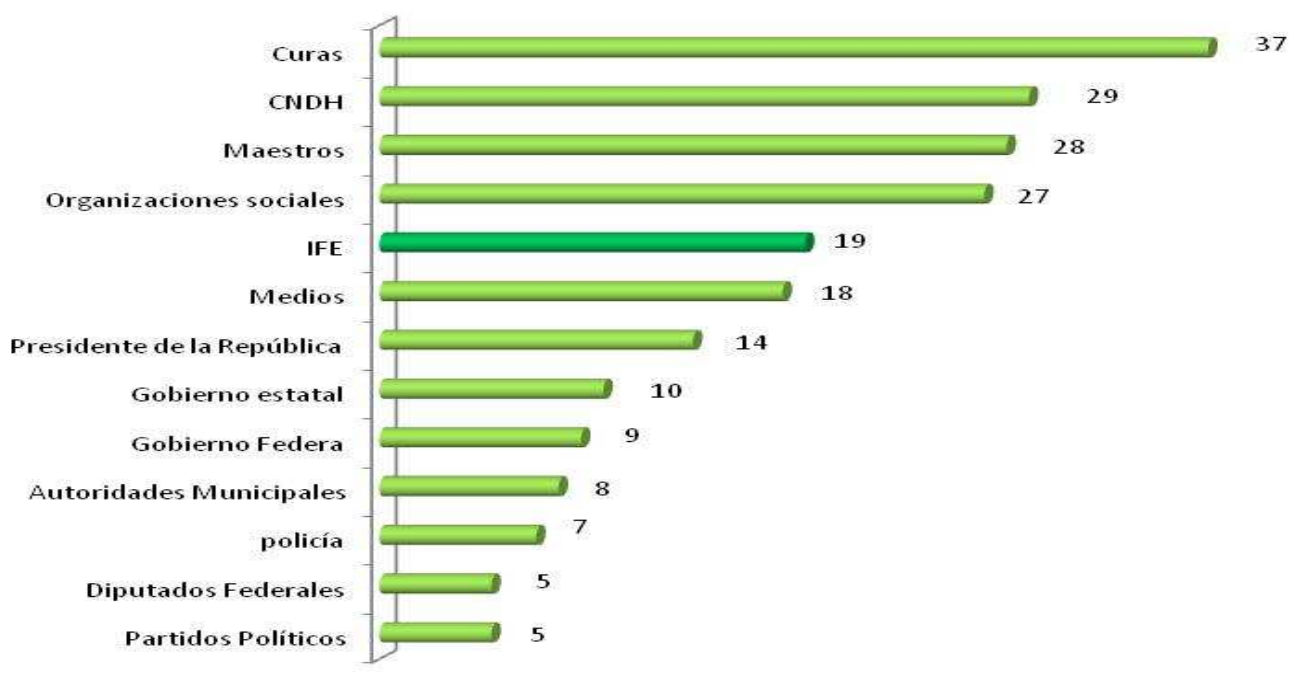

Fuente: Instituto de Investigaciones Sociales (2003).

Gráfica 4 - IFE: Confianza ciudadana, $2007^{5}(\%)$.

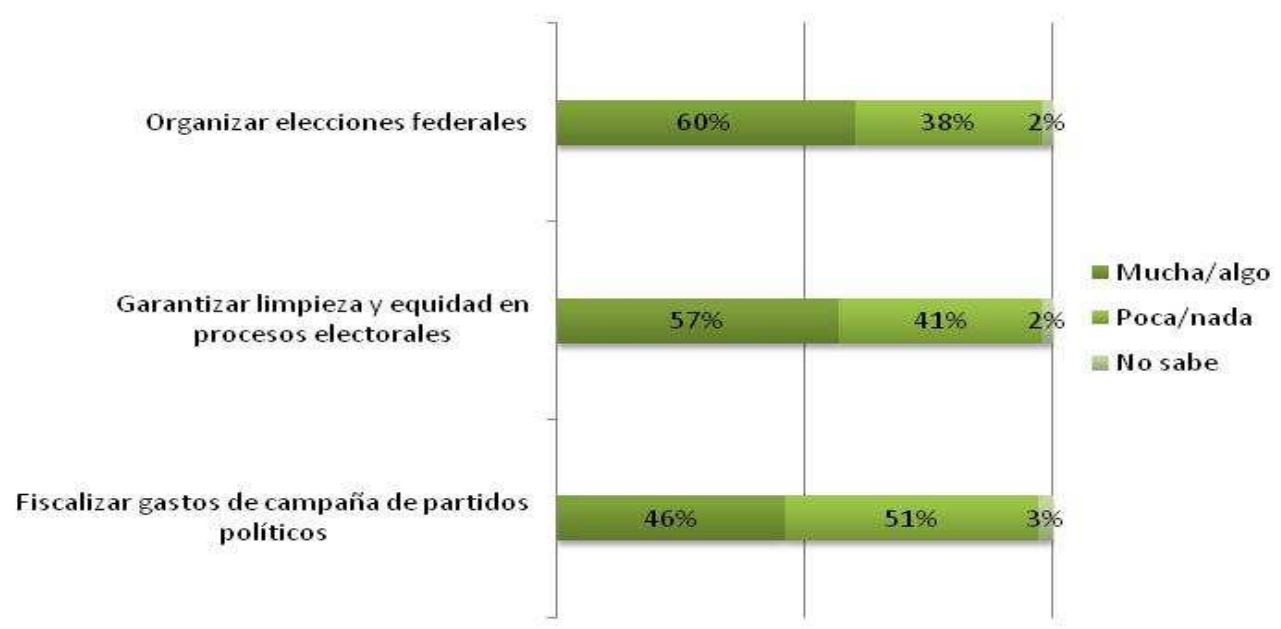

Fuente: Reforma (2007).

\footnotetext{
${ }^{4}$ Pregunta: En una escala en donde 0 es nada y 10 es mucho, qué tanto cree en lo que dice(n).

${ }^{5}$ Pregunta: ¿Cuánta confianza tiene el IFE para...?
} 
Gráfica 5 - IFE: opinión ciudadana acerca del IFE 2003-20076 (\%).

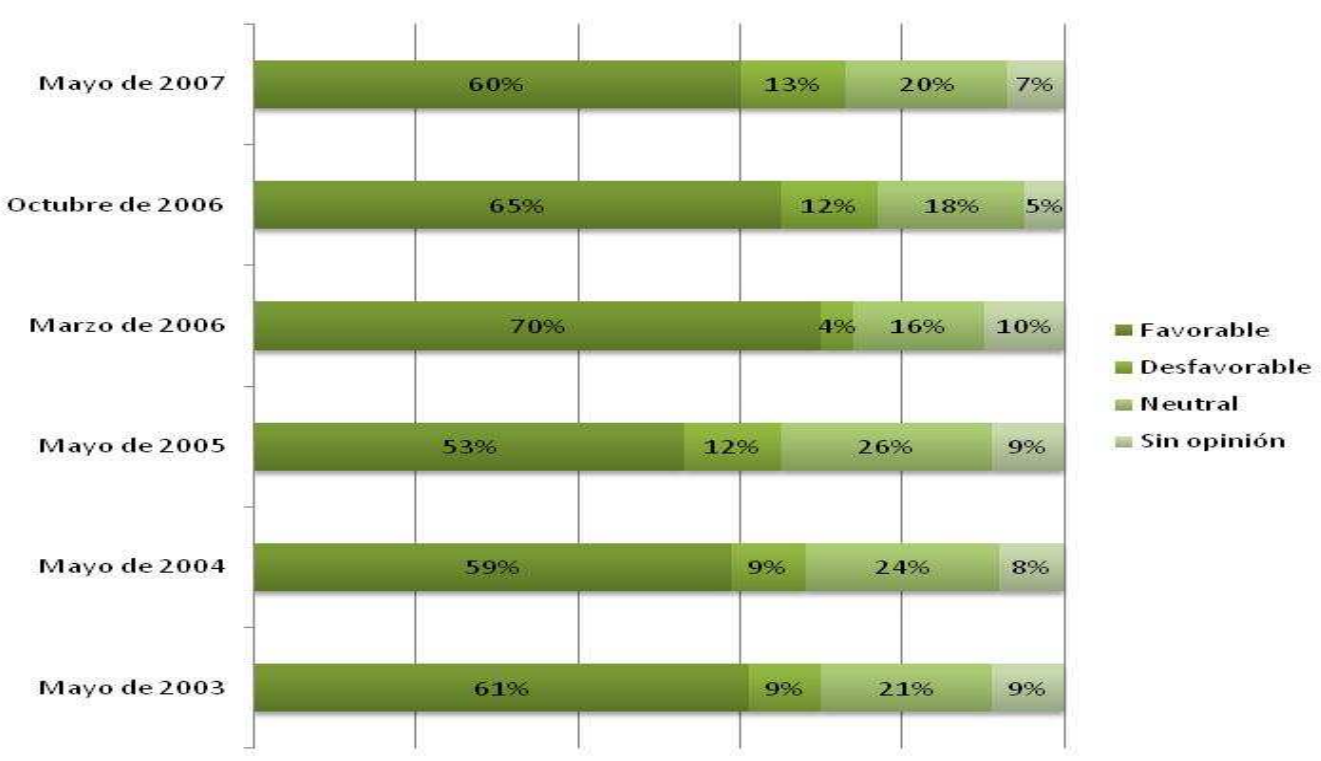

Fuente: Ipsos ${ }^{7}$.

Gráfica 6 - IFE: Confianza Ciudadana por entidades federativas, $2007^{8}(\%)$.

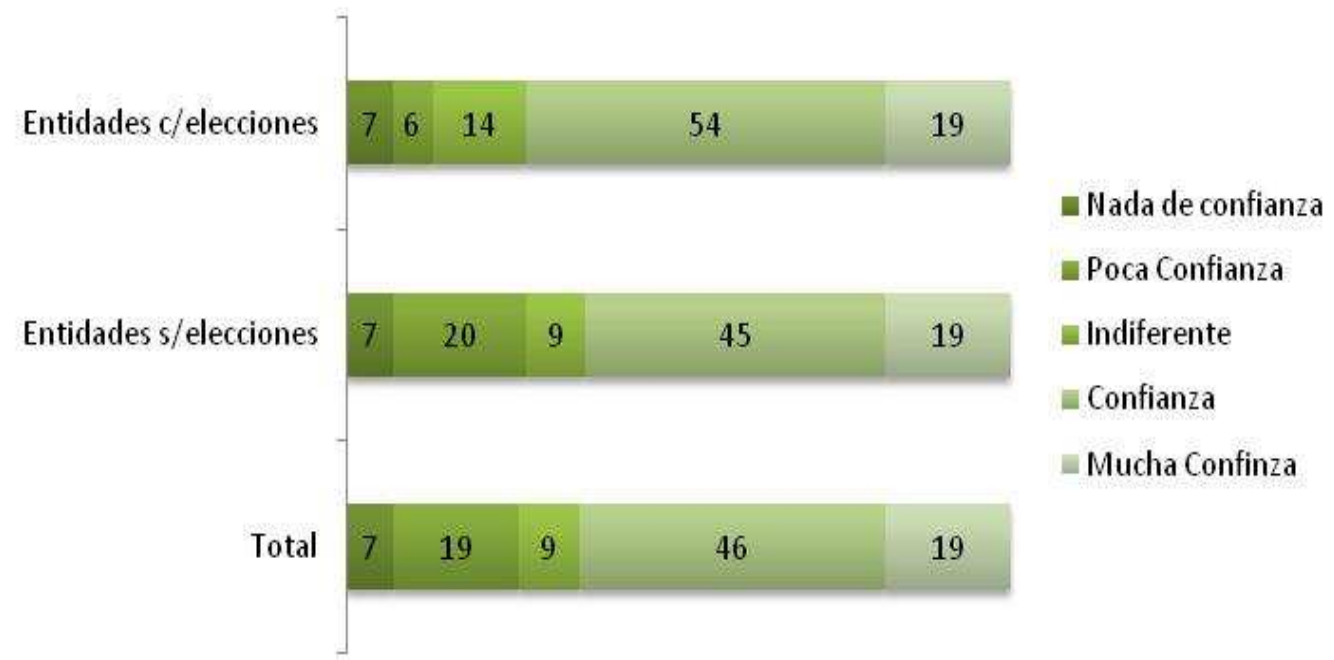

Fuente: Ipsos (2008).

${ }^{6}$ Pregunta: ¿Cuál es su opinión acerca del IFE?

7 Disponible en: <http://www.ipsos-bimsa.com.mx/>. Acceso em: 12 may. 2011.

${ }^{8}$ Pregunta: ¿Qué tanta confianza le tiene usted al Instituto Federal Electoral IFE?

REVISTA DEBATES, Porto Alegre, v.5, n.1, p. 9-23, jan.-jun. 2011. 
Gráfica 7 - México: confianza ciudadana en instituciones, $2010^{9}(\%)$.

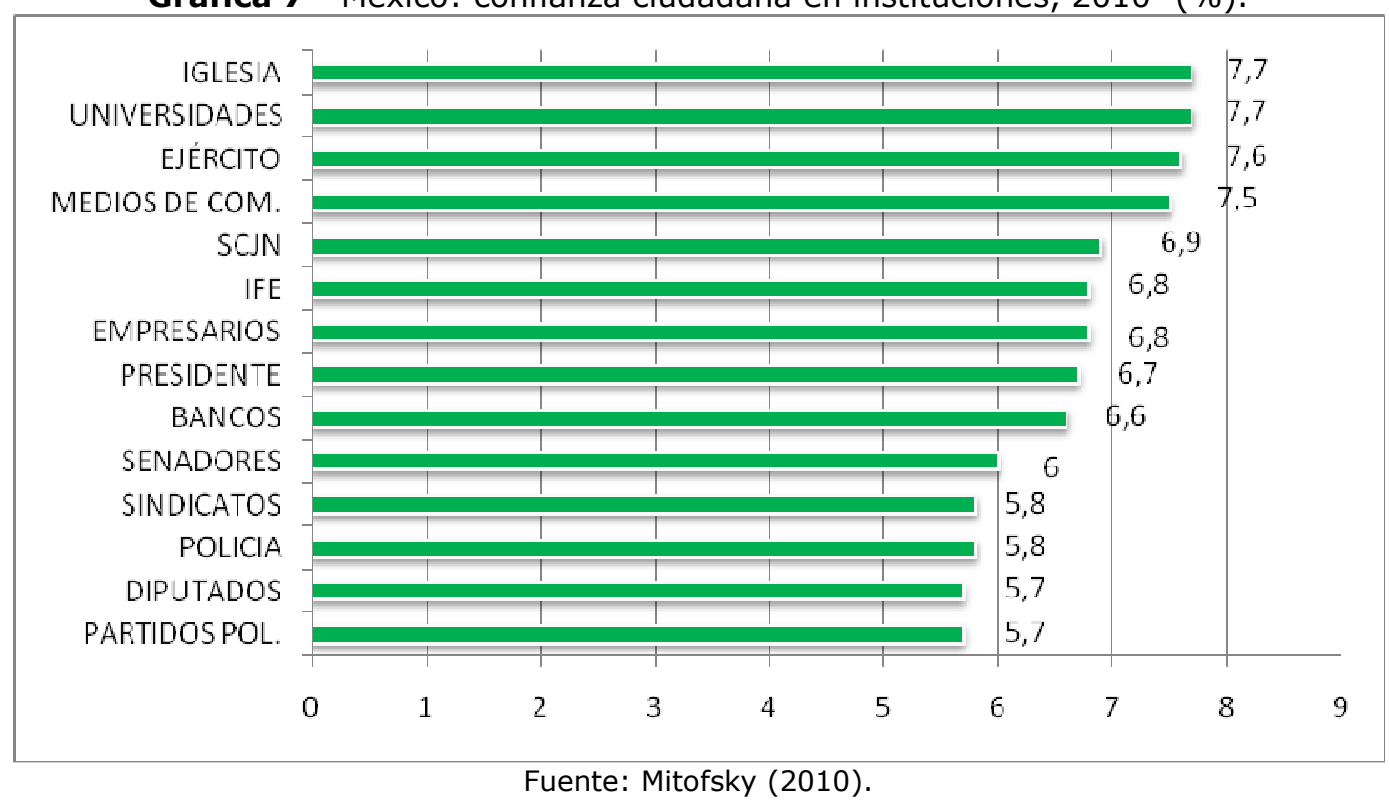

El análisis de todos estos datos arrojan las siguientes conclusiones:

- En el 2003 el $63 \%$ de la gente desconfiaba mucho o algo en el IFE, en el 2008 ya solo el $26 \%$ de la población desconfiaba mucho o algo en el instituto. Es decir hubo un repunte en la confianza, que otorga al organismo un mayor margen de maniobra en la toma de decisiones.

- En $61,3 \%$ es el promedio de opinión favorable entre 2003 y 2007. Con una sola cifra superior de $70 \%$ en marzo de 2006 , fecha previa al proceso electoral de ese año. Lo cual refleja cierto optimismo de la gente respecto a la actuación inminente del instituto.

- Entre 2003 y 2007 la opinión hacia el IFE se mantuvo en general como favorable en alrededor del $60 \%$, si bien aumentó la desfavorable del $9 \%$ al $13 \%$. Lo cual puede indicar un declive entre la gente que antes opinaba favorablemente y ha dejado de hacerlo. Las razones pueden estar en la actuación del 2006 y la tardanza en la emisión de los resultados electorales.

- Al evaluar a detalle las tareas del IFE la población le otorga menos confianza $(46 \%)$ en la fiscalización a los partidos políticos, en tanto una mayor confianza en organizar las elecciones y en la limpieza de los comicios. Ello puede estar indicando una baja credibilidad en los mecanismos de supervisión a los partidos políticos o una desconfianza en éstos.

\footnotetext{
${ }^{9}$ Pregunta: En una escala en donde 0 es nada y 10 es mucho, qué tanto confía en las siguientes instituciones.
} 
- Los acontecimientos políticos en que se ve involucrado el IFE no parecen afectar la opinión ciudadana, lo cual es una buena noticia, porque habla de la institucionalización del organismo.

- Respecto al conocimiento que la población tiene de las funciones del IFE los datos son contradictorios, según la empresa encuestadora (en general así sucede en casi todos los casos, de ahí la principal dificultad para el análisis histórico). En el año 2003 el 78\% reportaba que el IFE organizaba elecciones (PARAMETRÍA, 2003) y en 2008 solo el $36 \%$ reconocía que la principal función del instituto era organizar las elecciones (IPSOS, 2008).

- Cuando se examina la confianza en las entidades federativas ésta disminuye. Hacia el 2007 solo alcanza el 46\% de confianza. Puede tratarse de un alejamiento del IFE hacia las entidades federativas. Los acontecimientos políticos en que se ve involucrado el IFE no parecen afectar la opinión ciudadana, lo cual es una buena noticia, porque habla de la institucionalización del organismo.

- Los datos con que se cuenta son insuficientes para conocer la opinión de la gente respecto a la partidización del IFE. No se han hecho preguntas al respecto, por lo que dicho problema se ha quedado en los análisis más finos.

- En relación a la rendición de cuentas tampoco aparecen datos, de manera que no es posible conocer hasta que punto la gente considera que el IFE debe hacerlo.

- En general la confianza en el IFE no ofrece datos alarmantes de desconfianza, aunque tampoco han aumentado lo necesario para apuntalar la democracia electoral. Las elecciones federales del 2012 volverán a ser una prueba de la solidez del organismo; no solo como arbitro electoral, sino como institución que aspira a ser independiente de la política partidaria actual en México.

Con respecto a la confianza interpersonal el panorama es distinto, con respecto a la confianza interpersonal, la cual representa una base para la formación de capital social, que como se explicó da solidez, estabilidad y duración al sistema democrático. En la confianza interpersonal México está a mitad de camino, con relación al resto de los países del continente. Aquí si falta mucho por hacer, y se vislumbra un camino largo. Es posible afirmar, con base en los datos reportados por LAPOP que la confianza que va formando y fortaleciendo el tejido social, no es una realidad generalizada en México. Esta no es una buena noticia para una democracia de calidad, al menos en el corto plazo. Y lo que si puede ser alarmante es la formación de redes dentro del crimen organizado o el narcotráfico, que tanto poder han desarrollado en el país. Se perciben como redes sociales de efectos altamente peligrosos, es una forma de capital social poco deseable en una democracia en construcción.

Leticia Heras Gómez es Doctora en Ciencias Sociales por la Universidad Iberoamericana en México, Maestra en Latin American Politics and Government por The University of Essex en el Reino Unido, Maestra em 
Ciencia Política por la UAEM em México. Actualmente es profesora e investigadora de la Universidad Autónoma del Estado de México, y sus temas de investigación son cultura política, participación política.

E-mail: leticiaheras@hotmail.com

\section{Referências}

COLEMAN, James S. Social capital in the creation of human capital. Supplement: Organizations and Institutions - Sociological and Economic Approaches to the Analysis of Social Structure. The American Journal of Sociology, Chicago, v. 94, p. S95-S120, 1988.

Foundation of Social Theory. Boston: The Belknap Press of Harvard University Press, 1990.

EMV. Encuesta Mundial de Valores. 2008. Disponible en: <http://www.worldvaluessurvey.org/>. Acceso en: 12 may. 2011.

HERAS, Leticia. Rendición de cuentas en los organismos electorales: tarea inacabada en el procesos de democratización en México. 1. ed. Toluca, Méx.: IEEM, 2008. (Serie de Breviarios de Cultura Política Democrática, 6).

INGLEHART, Ronald. Cultura política y democracia estable. Revista Española de Investigaciones Sociológicas, Madrid, v. 42, n. 88, p. 45-65, abr.-jun. 1988.

INGLEHART, Ronald; WELZEL, Christian. Modernization, Cultural Change, and Democracy: The Human Development Sequence. New York: Cambridge University Press, 2005.

INSTITUTO de Investigaciones Sociales. UNAM, México, 2003.

IPSOS MARKETING RESEARCH. Reporte de resultados. México, D. F.: IPSOS BIMSA, Dec. 2008. Disponible en: <http://www.ife.org.mx/docs/IFEV2/DECEYEC/DECEYEC/-MateriaAudiovisula/DECEYEC-

SpotsIFE/EvaluaciónCuantitativa_CampaniaInstitucional2008-

docs/Reporte_de_Resultados_IFE_Camp_Inst_2008.pdf>. Acceso en: 12 may. 2011.

LAPOP. Latin American Public Opinion Project. Cultura política de la democracia en México, 2008. Impacto de la governabilidad. 2008.

Disponible en: <http://www.vanderbilt.edu/lapop/Mexico/2008impactodegobernabilidad.pdf>. Acceso en: 12 may. 2011. 
LIN, Nan. Social Capital: A Theory of Social Structure and Action. New York: Cambridge University Press, 2001.

LUJÁN PONCE, Noemí. La constitución de confianza política. México: IFE, 2009. (Temas de la democracia, 6).

MILLÁN, René; GORDÓN, Sara. El capital social: una lectura en tres perspectivas clásicas. Revista Mexicana de Sociología, Ciudad de México, año 55, n. 4, p. 711-747, oct. 2004.

MITOFSKY. Confianza en las Instituciones (Feb/10) - encuesta nacional en viviendas. 2010. Disponible en: <http://consultamitofsky.org>. Acceso en: 12 may. 2011.

PARAMETRÍA. Conserva IFE confianza ciudadana. 2003. Disponible en: <http://www.parametria.com.mx/DetalleEstudio.php?E=4246>. Acceso en: 12 may. 2011.

PUTNAM, Robert D. Making democracy work: civic traditions in modern Italy. Princeton: Princeton University Press, 1993.

Bowling Alone: The Collapse and Revival of American Community. New York: Simon and Schuster, 2000.

. Democracies in Flux: The Evolution of Social Capital in Contemporary Society. New York: Oxford University Press Inc., 2004.

REFORMA. Encuesta - Desconfían de fiscalización. Periódico Reforma, México D. F., 6 jun. 2007.

SEGOB. Secretaría de Gobernación. Encuesta Nacional sobre Cultura Política y Prácticas Ciudadanas. Ciudad de México: SEGOB, 2001.

- Encuesta Nacional sobre Cultura Política y Prácticas Ciudadanas. Ciudad de México: SEGOB, 2003.

. Encuesta Nacional sobre Cultura Política y Prácticas Ciudadanas. Ciudad de México: SEGOB, 2005.

Encuesta Nacional sobre Cultura Política y Prácticas Ciudadanas. Ciudad de México: SEGOB, 2008. 